previous winter, for only when conditions favour overwintering out of doors are the numbers of aphids large. The importance of the method of overwintering is also shown by Aphis rhamni, which in this region is found in numbers only in the few localities where the winter host Rhamnus catharticus occurs.

In East Anglia conditions are very different. Mr. J. P. Doncaster finds that A. rhamni is the commonest aphid on potatoes, and frequently reaches an average of more than a hundred per leaf. Fortunately, this aphid does not seem to transmit leaf roll. As it moves about less readily than Myzus persica, it is probably less important as a vector, but it is likely that the migratory forms play a considerable part in the transmission of potato virus $Y$. Overwintering on Brassicæ seems to be rare in East Anglia, but here there are sufficient peach trees and glasshouses to provide ample sources for $M$. persicae in the spring. No attempts have yet been made to control the aphids by treating their overwintering sources, for example, by wholesale winter-washing of trees and fumigation of glasshouses; but Mr. Doncaster reported that on the potato crop a complete kill was obtained by fumigation with nicotine. The effects of the treatment, however, were only temporary, and the fumigated crop rapidly became re-infested from nearby sources not treated.

After examining many potato crops in eastern England, Dr. P. H. Gregory and Mr. Doncaster could find no simple correlation between numbers of aphids and amount of spread of leaf roll and potato virus $Y$. It seems likely that in these districts there are almost always sufficient aphids for rapid spread and that whether this occurs or not depends on other factors, probably those influencing the movement of the insects. In many crops in 1941 there was a striking difference between the behaviour of the two aphidtransmitted viruses. Potato virus $Y$ spread only to a small extent and only early in the season, again suggesting a correlation between the winged migrants and spread of this virus. By contrast, the number of plants infected with leaf roll virus increased steadily throughout the season, and in some stocks with large haulms, in which movement of erawling aphids from plant to plant would be facilitated, the percentage of infected plants increased more than twenty times.

The ability of one and the same potato virus to produce different symptoms in different potato varieties greatly complicates diagnosis, but can also be of value in helping to reduce losses. Paradoxically, it is the most susceptible varieties that suffer least. Some British varieties, for example, King Edward and Epicure, die quickly when infected with virus $X$. Infected plants leave no viable tubers to act as further sources of infection, and the stock as a whole is safeguarded by the death of the individual. Similarly, other varieties are killed by infection with viruses $A, B$ and $C$. In effect, such varieties do their own roguing, and Dr. G. Cockerham showed that the health of commercial stocks of these varieties is much better than that of those which tolerate infection. This hypersensitivity is inherited, and by crossing between different varieties, the Scottish Society for Research in Plant Breeding has produced Craig's Defiance, which dies when infected with any of viruses $A, B$, $C$ and $X$, so that stocks of this variety remain virtually free from all four. Unfortunately, no varieties are known which are hypersensitive to leaf roll and virus $Y$, but after testing some hundreds of different varieties Dr. Cockerham reported that he had found some with a true resistance. As this is also inherited, it seems that the geneticist will begin to play an increasingly important part in combating potato virus diseases. However, no quick success can be expected by this method; also, viruses appear to mutate with such frequency that permanent success should not be expected either, as it is impossible to be sure for how long a new variety bred for some specific property will retain this in the field.

In an attempt to increase the health of crops from once-grown seed, the Ministry of Agriculture has since the War introduced a scheme whereby crops in England, grown from Scotch or Irish seed and planted fifty yards from other potatoes, receive a seed certificate. Mr. Samuel showed that the measure is meeting with some success, for such seed usually contains less than 10 per cent of severely affected plants and crops as well as new seed, but it does not greatly increase the useful life of the stock. One wonders whether the information we now have about potato virus diseases could not be still further applied. In normal times it may be that the import of new seed each year is the most economical control measure, but now the transport of 300,000 tons of potatoes is in itself a problem. In many parts of England it is likely that the growing of heavy crops of ware potatoes and the production of good seed are incompatible, for maximum yield is obtained only if the plants have a long growing period and this greatly increases the likelihood of infection. But crops grown especially for seed purposes could probably be kept in a good state of health for several years. These could be grown in isolation, possibly on some of the reclaimed land that cannot be expected to yield large erops, rogued, perhaps sprayed or fumigated against aphids, and the tops killed early in August, after all the tubers are formed but before virus diseases have had time to spread appreciably. The contributions at the discussion all suggested that this may be a practical control measure, but it urgently needs testing on a commercial scale.

\section{VELOCITY OF RADIO-FREQUENCY WAVES*}

\section{By DR. R. L. SMITH-ROSE \\ National Physical Laboratory}

$T$ $\mathrm{HE}$ wireless engineer and radio physicist require to know the speed or velocity of electric waves in order, for example, to convert their measurements of frequency into the corresponding wave-lengths, while various methods of studying the propagation and direction of arrival of waves involve a knowledge of the wave velocity.

Apart from the various classical experiments on the velocity of light conducted during the latter half of the nineteenth century, the most comprehensive work on this subject was carried out by Prof. Michelson and his co-workers, and the most important part of these investigations were in progress when Michelson died in 1931. The equipment used in this work is notable by reason of the fact that the path traversed by the light waves was enclosed in a steel tube one mile long and three feet in diameter,

* Substance of the chairman's address before the Wireless Section of the Institution of Electrical Engineers, delivered on Oct. 7 . 
this tube being evacuated to a pressure of a few millimetres of mercury. By multiple reflexions between mirrors set up in this tube, the light waves were caused to traverse a total path-length of some 8-10 miles in a vacuum before and after reflexion from a thirty-two-faced rotating mirror used to determine the time of travel of the waves over this path.

The development of radio-frequency technique has facilitated the measurement of the velocity of light on a laboratory scale by a method which has been used by W. C. Anderson and others. In this, the light waves are modulated at a radio-frequency of from 20 to 100 megacycles per cycle by their passage through a Kerr cell subjected to an alternating voltage at this frequency. Two portions of this modulated light beam are made to traverse paths differing in length by some 10-20 metres before being received on a photo-electric cell equipment used to measure the resulting intensity of the light received.

A summary of the most accurate measurements carried out by these two methods is given in the following table:

Reoknt Measurements of the Velocity of Light

Values refer to transmission in a vacuum

\begin{tabular}{|c|c|c|c|c|}
\hline Date & Observers & $\begin{array}{c}\text { Velocity in } \\
\text { cm. per sec. } \\
\times 10^{10}\end{array}$ & $\begin{array}{c}\text { Accuracy of } \\
\text { measurement }\end{array}$ & $\begin{array}{c}\text { Difference } \\
\text { from } \\
c=3 \times 10^{10}\end{array}$ \\
\hline $1931-33$ & $\begin{array}{c}\text { Michelson, } \\
\text { Pease and } \\
\text { Pearson } \\
\text { Anderson }\end{array}$ & 2.99774 & 0.4 in $10^{4}$ & -7.5 in $10^{4}$ \\
$1936-40$ & 2.99776 & 0.5 in $10^{4}$ & -7.5 in $10^{4}$ \\
\hline
\end{tabular}

Since for radio purposes we are rarely concerned with wave transmission in a vacuum, the above values will require to be multiplied by $1 / \sqrt{ } \kappa$ where $\kappa$ is the dielectric constant of the medium in question. For air under normal atmospheric conditions at the earth's surface in Great Britain, corresponding to a pressure of $760 \mathrm{~mm}$. mercury, temperature of $15^{\circ} \mathrm{C}$. and a relative humidity of 70 per cent, the value of $k$ is about $1 \cdot 0007$, and thus the corresponding value of the velocity will be reduced to $2.99670 \times 10^{10} \mathrm{~cm}$. per second.

Our knowledge of the speed at which waves in the radio-frequency spectrum travel is much more limited and less precise. Until about 1935, most investigators in this field used methods which were not free from sources of error arising either from defects in the apparatus or from an insufficient knowledge of the actual path followed by the waves. More recently, some workers have used methods involving the setting up of standing waves in air or on wires, and compared the wave-length so obtained with the frequency of the oscillations given by the source. In 1937, W. Ross and E. C. Slow, of the Radio Department of the National Physical Laboratory, made a determination of the phase velocity of radio waves transmitted along the surface of the ground, using apparatus already developed and in use for other investigations. The average result obtained for this velocity over the wave-length range 20-120 metres $(2 \cdot 5-15 \mathrm{Mc}$./s.) was $2.95 \times$ $10^{10} \mathrm{~cm}$./sec., the overall experimental accuracy of the measurements being considered to be about 5 per cent.

A most comprehensive investigation of the velocity of medium radio waves (wave-lengths $230-345 \mathrm{~m}$.) was carried out during the years 1934-35 by a group of workers in the laboratories of the U.S.S.R. under the direction of Profs. L. Mandelstam and N. Papalexi ; and the details and results of these investigations were published in a collection of papers in Technical Physics of the U.S.S.R. in 1937. The method employed comprises a determination of the time of transit of waves between a sending and reflecting station by measuring the phase difference between the two sets of waves, the ratio of the frequencies of which is rational. The subsidiary investigations included the development of methods and instruments for measuring the phase difference between harmonic oscillations of different frequencies, and the study of the phase displacement introduced by high-frequency amplifiers. The accuracy of the measurements depends essentially on the measurement of the two quantities, the phase displacement, and the length of path traversed by the waves between the stations. The total phase displacement used during the observations ranged from a few hundred to several thousand degrees, and the investigators were able to measure these to within $3^{\circ}$ or $4^{\circ}$; so that the accuracy of phase measurement was of the order of one or two parts in a thousand in the most favourable cases. The distance measurements were carried out by standard geodetic survey methods, and appear in the most favourable cases to have been determined to an accuracy somewhat better than one part in a thousand $(0 \cdot 1$ per cent).

The mean results obtained in the various sets of experiments for the velocity of the medium radio waves used all lie within the limits of $2 \cdot 990$ and $2.995 \times 10^{10} \mathrm{~cm}$./sec. for transmission over a clear air path, or over sea or fresh water. As these limits are within the accuracy of the measurements, no definite conclusions can be drawn as to the effect of a salt- or freshwater path on the velocity of propagation. In the case of transmission overland, a somewhat lower value of $2.950 \times 10^{10} \mathrm{~cm}$. $/ \mathrm{sec}$. was obtained, although this would appear to be subject to some uncertainty in determining the true path of transmission, due to various intervening obstacles.

Thus it is found that for the radio-frequency portion of the spectrum, the methods so far used for the determination of the velocity are much less accurate than those used for the velocity of light and it may be stated that within the limits of the experimental accuracy so far attained, which is not better than $0 \cdot 1$ per cent, the velocity of propagation of medium radio waves is about $2.990 \times 10^{10} \mathrm{~cm}$. $/ \mathrm{sec}$. The experimental evidence so far available indicates that this value for the velocity is the same for all radio wave-lengths within the accuracy specified above. There is some evidence that the phase velocity may be reduced below this value near the transmitting aerial, and it may also be modified when transmission takes place close to the surface of the earth; but it seems doubtful if this variation from the free space value amounts to more than the experimental accuracy, except where obstacles such as trees and mountains intervene in the path of propagation. In this case it is not necessarily the velocity which has altered, but rather that the path traversed has been increased.

This discussion of our present knowledge leaves the problem of the measurement or calculation of the actual time of transit of radio waves between sender and receiver still open to investigation. This type of information may be required for a number of purposes, such as the use of time signals for the comparison of chronological standards and for the 
determination of differences of longitude. For the solution of this problem, a knowledge of the free space speed is only one requirement : it still needs a precise specification of the actual path, rectilinear or otherwise, followed by the waves, and of any modification to the velocity value produced by atmospheric or ionospheric conditions.

\section{MICROBIAL FACTORS IN THE DIGESTIVE ASSIMILATION OF STARCH AND CELLULOSE IN HERBIVORA}

\author{
By FRANK BAKER \\ County Technical College, Guildford
}

$\mathrm{M}$ ICROBIAL processes are directly concerned in the digestive assimilation by Herbivora of the starch and crude fibre components of their diet. In ruminants the rumen and in non-ruminants the cæcum is the chief focus of these activities. Great diversity of opinion, however, exists in regard to the type or types of micro-organisms primarily responsible for the changes taking place ${ }^{1,2}$. This confusion must in large measure be attributed to the application of methods inappropriate to the resolution of the problem; and in particular to the exclusive and premature reliance placed on pure cultural technique. For, first, it is impossible by plating out to distinguish with certainty between the casual passengers through and the permanent inhabitants of the gastrointestinal tract. Secondly, the majority of micro. organisms indigenous to the cæcum and rumen are not susceptible of cultivation on any of the media ordinarily employed.

I have shown, however, that these difficulties can be circumvented by the use of methods of direct microscopical observation ${ }^{3}$. It must be emphasized that these methods are not confined to a mere academic enumeration of species. By examination in polarized light $t^{3,4,5,6}$ the disintegration of cellulosic structures can be studied in detail from start to finish in material freshly withdrawn from the rumen or creum. The changes taking place can be still further elucidated by histochemical and staining procedures ${ }^{4,5,7}$. Similar methods can be applied to the study of starch granules in process of disintegra. tion. Thus the functional activities as well as the morphological characteristics of the micro-organisms present may be ascertained. The results obtained can be recorded by photomicrographs. Nor, again, is the method of direct microscopical observation limited to qualitative data. Ferber long ago showed that the influence of dietetic factors on the rate of multiplication of the rumen Protozoa could be studied by the use of counting methods ${ }^{8,1}$. In collaboration with the Hannah Dairy Research Institute I have since found that similar methods can be extended to the iodophile microflora of the rumen and cæcum. Lastly, it may be noted that the method may be applied to formalized samples, so that material may be preserved in vitro for an indefinite period. This makes it possible to check or amplify observations over the entire course of a lengthy investigation. Similarly, by the use of coded samples, the objectivity of the data obtained by counting methods can be placed beyond question and subjective influences eliminated.

It is clear, therefore, that by the systematic assemblage and examination of appropriate collections of material a whole field of research is opened up which lies altogether beyond the range of pure cultural methods of investigation. These possibilities will, however, only be realized to the full when the method of direct microscopic examination has been adopted as a routine procedure by a sufficient number of observers. Nevertheless, observations accumulated over the course of several years already make it possible to elucidate some of the more conspicuous features which attend the microbial decomposition of starch and cellulose in Herbivora. The purpose of this article is to outline a consistent picture of these features and tentatively to indicate their significance in regard to the digestive process of the host-animals. To this end it is necessary briefly to summarize the general characteristies of the microbial associations established in the rumen or cæcum. These associations include, in addition to a number of species with which we need not here concern ourselves, ${ }^{4,5,9}$ :

(a) Iodophile micro-organisms of taxonomically diverse types such as collectively constitute the iodophile microflor ${ }^{9}$;

(b) Protozoa belonging to a highly specialized order of ciliates, the Entodiniomorpha11.

Both these groups play a part in the carbohydrate transformations of the gastro-intestinal tract. The distinctive feature of the iodophile micro-organisms is the blue-black reaction which they give with iodine, owing to the deposition within them of a polysaccharide ${ }^{2}$, the so-called bacterial starch or granulose. Most of the species encountered are of unusually large dimensions $\mathrm{s}^{4,5,7,9,10,13}$. They include sarcina ${ }^{5,13}$, Diplococcus ${ }^{7}$, Streptococcus ${ }^{4}, 5$, $7,9,13$, Spirillum ${ }^{5,13}$, and Clostridial forms ${ }^{10}$, together with sporing and non-sporing rods $7,9,10$ and several species of uncertain systematic position ${ }^{5,13}$. In addition, organisms of Oscillarian affinities are encountered in the guinea pig, sheep and $\operatorname{cow}^{9,12,13}$.

The iodophile microflora becomes established in the rabbit towards the close of milk feeding with the adoption of a vegetable diet ${ }^{14}$. Different constellations of species may be present in different species of Herbivora. Where these differences are marked, as in the case of the guinea pig and rabbit, they may persist even when the animals are fed on the same diet and kept in the same cage. Thus internal as well as external factors determined the characteristics of the microflora.

That certain iodophile species play a determinate role in the decomposition of cellulose can be demonstrated by direct microscopic examination in polarized light ${ }^{6}$. The iodophile micro-organisms are encountered upon the surfaces and within the interstices of the vegetable fragments present. Disintegration is accompanied by excision of enzymatic cavities, the contour of which corresponds precisely with that of the micro-organisms by which they are produced. In the affected regions birefringence ceases and microchemical reactions disappear. Lignified structures partially and cutinized structures entirely resist disintegration. The age, therefore, as well as the botanical species of the plant material fed will affect the amount of cellulose digested ${ }^{3}$.

Examination of rumen or cæcal contents demonstrates that iodophile micro-organisms are also directly concerned in the decomposition of starch. 